$5-7$

\title{
BMP Debate: evidence in the name of science?
}

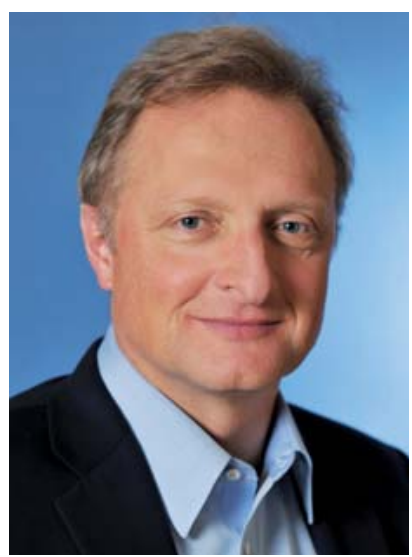

Jens Chapman
Spine surgery and spine surgeons have become attractive magnets for media attention... unfortunately, often the wrong kind of media attention [1, 2]. The recently unfolding debate among some of the biggest academic names in North American spine surgery touches on the subjects of recombinant human bone morphogenic protein-2 (Infuse ${ }^{\mathrm{TM}}$, Medtronic, Memphis, TN), off-label use of implants, conflict of interest, and expectations regarding financial disclosure and has expanded into a public mudslinging using the North American Spine Society's journal The Spine Journal (E Carragee, editor-in-chief) as its primary vehicle with many news outlets having jumped onboard [3-5]. Let's take a brief look at the two primary issues at stake here: (1) the actual science in dispute; (2) the issue of academic and research integrity.

Actual science: At its core, a study by Carragee et al [3] described a higher rate of retrograde ejaculation (RE) in male patients in a personal single-surgeon series following anterior lumbar interbody fusion with rhBMP-2 compared with a historical control group using the same interbody device without rhBMP-2. The authors reported 5 of 69 patients in the rhBMP-2 cohort to have RE compared with 1 in 174 patients in the control group. Three patients with RE in the rhBMP-2 group were found to have osteolysis around their allograft ring, with at least one patient having experienced a fracture of the upper end plate of S-1. Also, at 1 year, the authors identified that three of the total of six patients with RE had spontaneous resolution of their symptoms (two in the rhBMP-2 group, one in the control group). Two of the 'older' (sic) patients in the rhBMP-2 group (48- and 53-year-old patients) did not recover. This latter statement is a bit confusing-did three of the rhBMP-2 patients recover by 2 years, or just two? The third patient in question is not addressed.

From a methodological point of view, the number of patients with RE at the conclusion of 2 years, as reported, is important. If it is indeed 3 of 69 patients in the BMP study group and 0 of 174 in the historic control group, then the $P$ value in our calculation would be .02 (two-sided Fisher exact test), suggesting a statistically significant difference. However, if 2 of 69 patients are left with permanent RE, with 0 of 174 patients in the control group, the $P$ value is .08 (two-sided Fisher exact test)—NOT significant! Either way, claims based on "statistical significance" hinging on a single patient reflects poor judgment. There are other variables not addressed, such as type of anterior hardware used and dosage of rhBMP-2, as well as a number of other variables. The very small numbers regarding RE hardly provide scientific grounds to support the authors' conclusion of "strongly suggesting rhBMP-2 use with an anterior interbody fusion at the lumbosacral junction is associated with an increased risk of RE."

Note that the carrier vehicle chosen in the study of Carragee et al is indeed an off-label device, and that the US Food and Drug Administration study with rhBMP-2 was performed with tapered threaded titanium cages, which are not subject to the osteolysis-discussed by Carragee and colleagues to be possibly causative in the complications. The emerging 
subject of allograft osteolysis is a fascinating and unexpected recent discovery from the use of rhBMP-2-an observation that once solved could help us understand bone biology much better. Regarding these circumstances the wording "potentially statistically significant" in the conclusion of Carragee et al might seem preferable.

The subject of RE is certainly an important discussion point to cover with male patients before any lumbosacral surgery, but does not address the larger subject of sexual dysfunction after anterior lumbar spine fusion surgery. In their study Berg et al [6] identified $26 \%$ of males in an anterior lumbosacral fusion control group without BMP (compared with 3\% in their disc replacement study group). From a scientific point of view, Carragee et al deserve credit for bringing the subject of sexual dysfunction surrounding spine surgery to the forefront and looking at variables that may lead us to better understand factors that play into this. What has been completely lost in this debate is the adverse role that low back pain (LBP) plays in the sex life of both genders (in the Berg et al study, 34\% of patients reported that their sex life caused extra LBP and 30\% reported that their sex life was severely impaired by LBP.) The authors found an improvement of sex life correlating to a reduction of LBP following surgery either with fusion or lumbar disc replacement [6].

\section{Academic integrity?}

Complications related to BMP-2 have been reported in publications before the Carragee et al study, and in fact some reported complications were found to be high. A special focus issue of Spine in April 2010 (This focus issue was commissioned by AOSpine North America, a regional subsidiary of AOSpine International, which publishes EBSJ together with the AO Foundation) featured a high-quality, comprehensive systematic review of 31 studies describing complications related to use of osteobiologics in spine surgery [7]. In a systematic review of complications related to rhBMP-2 use in spine surgery, Mroz et al [7] found an overall mean complication rate of up to $8.4 \%$ (range, $0 \%-100 \%$ ) across various types of complications. Of these the highest mean rates across multiple studies were: resporption/osteolysis (vertebrae, graft, or end plate) following lumbar spine surgery, 44\% (range, $0 \%-100 \%$ ) and following cervical fusion, $43 \%$ (range, $0 \%-100 \%$ ); graft subsidence, $43 \%$ (range, $41 \%-44 \%$ ) in the cervical spine and $25 \%(0 \%-62 \%)$ in the lumbar spine; cage migration (lumbar), 27\% (0\%-35\%); extradiscal, ectopic, or heterotopic ossification, $8 \%$ (range, $0 \%-75 \%$ ) in the lumbar spine and 3\% (range, $2 \%-13 \%$ ) in the cervical spine. The subject of RE did not reach a critical threshold in this very frank review by these authors. The accusations of underreporting complications, however, are hard to uphold given these published complication rates.

As this example may show, data are subject to interpretation. An article, which raises truly foundational questions of side-effects of rhBMP-2 was not picked up by news media. Instead, an article with far less statistical power to find a real difference-if it actually exists (see discussion above on the Carragee's article)-received substantial media play. Why is that? The resonance to the Carragee's article receives particular poignancy when seen in the context of an ongoing US government agency and Senate investigation of potential conflicts of interest surrounding spine surgery and potential adverse implications on academic integrity in the underreporting of complications. The greater allegation is that serious complications regarding rhBMP-2 use in spine surgery may have been purposely left out of key publications on index RCTs and that financial ties to the manufacturer may have influenced what evidence is published and how it is 


\section{References}

1. Moore J (2011) Study links Medtronic product to sterility risk. StarTribune.com. Available at: www.m.startribune. com/news/?id=122631004.

2. Carryrou J (2011) Senators look into Medtronic, doctors. Wall Street J. Available at: www.wsj.com/article/SB1 00014240527023040701045 76400032473761332.html.

3. Carragee EJ, Hurwitz EL, Weiner BK (2011) A critical review of recombinant human bone morphogenetic protein-2 trials in spinal surgery: emerging safety concerns and lessons learned. Spine J; 11(6):471-491.

4. Zdeblick TA (2011) Science please. Spine J; 11(7):686. Epub 2011 Jul 20.

5. Carragee EJ, Hurwitz EL, Weiner BK (2011) Authors and editors combined response to Zdeblick letter (revised 28 June 2011). Spine J; 11(7):687690. Epub 2011 Jul 20.

6. Berg S, Fritzell P, Tropp H (2009) Sex life and sexual function in men and women before and after total disc replacement compared with posterior lumbar fusion. Spine J; 9(12):987-994. Epub 2009 Oct 12.

7. Mroz TE, Wang JC, Hashimoto $R$, et al (2010) Complications related to osteobiologics use in spine surgery: a systematic review. 35(9 Suppl):S86-104.

8. Gary Schwitzer's Health Blog: (2006) www.healthnewsreview.org/blog/2011/06/ harm-has-been-done-spinejournals-critical-review-ofmedtronic-infuse-studies.html presented. When this happens, invariably we all lose, including our patients, and care decisions may be made not based upon best medical interests of our patients but by third parties with a different agenda. Such allegations leave a professional credibility gap for us that are difficult to close [8].

We rely on Academic honesty when we read the published research that we use to inform our patients of the benefits and risks of any treatment and make treatment suggestions and decisions. We trust that our colleagues who are reporting research are telling us the truth. Disclosures of background variables-such as financial arrangements or pressure applied by external third parties (eg, government agencies, third-party payors, publishers, and specialty societies) may allow readers to form their own opinion about the potential for bias. If disclosure does not occur it opens the door for inquiry and later sensationalism and trivialization in the public forum ("Male infertility caused by BMP!!!").

Undoubtedly we will hear more about this subject in the next months as lawyers and statisticians will take sides and accusations and revelations will be aired in a variety of public settings, and not in a collegial setting with frank scientific discussion aimed at advancing patient care. The actually interesting physiological discoveries of allograft osteolysis and possible radiculopathy emulated by inflammatory mediators following application of rhBMP-2 may get lost in the discourse-although it highly deserves to be evaluated in much greater detail.

EBSJ is dedicated to reporting the facts as clearly as possible. Our systematic reviews are created based on reader requests and emphasize presentation of the data in a transparent manner so that readers can draw their own conclusions. This is not glamorous or conducive to attracting media attention, but "shows the numbers" to allow us to do what we are ultimately supposed to do: to take care of our patient's best we can.

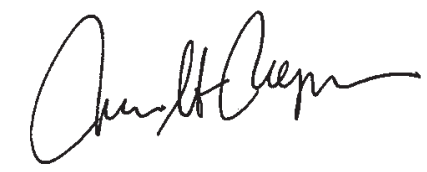

Jens Chapman

Editor-in-chief 